\title{
Polysèmes
}

Revue d'études intertextuelles et intermédiales

\section{Rouge : Victor Hugo ou la couleur en perspective}

Red: Victor Hugo or Color in Perspective

\section{Stéphanie Boulard}

\section{(2) OpenEdition}

Journals

Édition électronique

URL : http://journals.openedition.org/polysemes/1439

DOI : 10.4000/polysemes. 1439

ISSN : 2496-4212

Éditeur

SAIT

Référence électronique

Stéphanie Boulard, «Rouge : Victor Hugo ou la couleur en perspective », Polysèmes [En ligne], 14 | 2015, mis en ligne le 18 novembre 2015, consulté le 09 octobre 2020. URL : http://

journals.openedition.org/polysemes/1439; DOI : https://doi.org/10.4000/polysemes.1439

Ce document a été généré automatiquement le 9 octobre 2020.

Polysèmes 


\title{
Rouge : Victor Hugo ou la couleur en perspective
}

\author{
Red: Victor Hugo or Color in Perspective
}

Stéphanie Boulard

« Eh! N’oubliez pas le rouge!»

Baudelaire

1 «Nous n'avons que le choix du noir", écrit Victor Hugo dans William Shakespeare. Le choix du noir, c'est bien celui en effet que fait Victor Hugo tant dans ses dessins ou aquarelles, que dans ses écrits, utilisant dans les deux cas la même plume, la même encre, voire le même papier, plaçant l'encre au cœur de la dialectique ombre/lumière. Mer d'encre, bouche d'ombre, océan de noirceur, nombre de critiques auront donc noté cette prédominance accordée à l'ombre et aux ténèbres pour y lire chez Hugo une phénoménologie négative vis-à-vis du monde visible, les autres couleurs venant nécessairement s'établir en contraste ou au contraire du noir. Annie Le Brun l'aura bien vu: la présence du noir dans l'œuvre hugolienne peut se lire comme matière génératrice de lumière, transcendantale, l'arc-en-ciel d'une constante plongée dans les ténèbres d'où surgit l'étincelle, l'éclair, l'horizon, où est à l'œuvre ce qui embrase «le ciel intérieur " (Le Brun, 31). Car il ne fait aucun doute que, pour Victor Hugo, l'écrivain est un écrivain de la nuit et qu'il est le contempleur de l'ombre', celui qui fait avancer la poésie dans «l'opacité du mystère » (Millet, 310). Miser sur le noir, c'est miser sur la communication par l'abîme dont il faut explorer toutes les polysémies², tous les synonymes: ténèbres, obscurité, nuit, ombre. Lisons: la pensée flotte, libre, dans l'infini du rêve et de la nuit. Comme Shakespeare, Victor Hugo est le «semeur d'éblouissements ", conclut Annie Le Brun (127).

2 Or, si dès la Préface de Cromwell, Hugo sort la couleur de sa contingence et qu'il l'engage dans l'écriture qui vient ainsi faire un pendant aux dessins, ce n'est pas seulement pour en colorer son style, mais pour l'inscrire dans un système politique, symbolique et métaphorique complexe ${ }^{3}$. Pour Victor Hugo, en effet, l'idée $a$ une couleur. Et, plus précisément, et contrairement à ce qu'il affirmait plus haut: l'idée de rouge. Nous voulons démontrer que le rouge, chez Hugo, établit avec l'expérience de l'ombre non 
pas un contraste, mais une voie de passage ou une complémentarité. Le rouge, qui n'est pas la couleur dominante de ses dessins, envahit paradoxalement les pages de ses romans ou de ses cahiers de notes, et devient en tant que tel une vision haptique spectacle de théâtre, pôle d'attraction, signe politique, entourant surtout un objet entre tous, un centre de fascination absolu et terrible, j'ai nommé : la guillotine.

La guillotine, voilà bien un mot, un objet, qui aura fait couler beaucoup d'encre. On sait toute l'horreur et la fascination que suscite la machine à décapiter de la révolution, ce monstre de bois de justice, justement rouge. La présence insistante de la machine dans les romans hugoliens peut bien sûr être éclairée par l'émotion très profonde qu'elle provoque sur les contemporains de Hugo, le public en général, mais aussi les écrivains.

Or, disons-le d'emblée : ce n'est pas un objet parmi tant d'autres, et c'est toute l'œuvre hugolienne qui est traversée par la silhouette, le rêve ou le fantôme, de la guillotine. À commencer par le terrible dessin Justitia ${ }^{4}$. De là à dire que Hugo reconnaît la guillotine comme son objet, c'est la suggestion que je veux faire. Elle y est d'une massivité discrète mais obsédante, en ce qu'elle laisse dans l'œuvre une marque indélébile. On ne peut alors qu'être invité à suivre ainsi ce qu'on peut définir comme un fil rouge, dans toute la complexité de ses sens. Jusqu'au vertige... La plume plonge dans la rougeur sanglante de la violence et de la vie mêlées, du sang répandu et du sang qui vivifie. Et de fait, l'écriture puise à la source de l'effroi, d'où son intensité, son tranchant, et le "vif» de son insertion dans la «chair» des choses et des êtres ainsi suivis à la trace. C'est cette ambiguïté vitale du marqueur rouge, dans le tissage du texte, que nous voulons suivre.

\section{Rouge guillotine}

5 En vérité, la question de la guillotine, Hugo, très tôt, y a trempé sa plume, et on pourrait aller jusqu'à dire, le parodiant lui-même, «qu'il se l'était lui-même enfoncée dans les entrailles, et à cette heure, en $1[a]$ considérant, il pouvait avoir l'espèce de satisfaction qu'aurait un armurier qui reconnaîtrait sa marque de fabrique sur un couteau, en se le retirant tout fumant de la poitrine » (Hugo, Les Mis, 1089). Au sang versé par la guillotine, répond l'encre versée par l'écrivain qui y aura tellement aiguisé son style qu'une certaine Madame Trollope écrira ceci : « La France rougit en lisant les livres de Victor Hugo » (Hugo, Choses Vues, 1317). Il y a de quoi rougir en effet : la tache de sang qui s'étale sur les pavés de Paris contamine le corps écrit et vient teinter de rouge, rubriquer, les mots du poète. Rouge: couleur annoncée, affichée comme un étendard.

6 Et d'abord, comme le rouge, elle attire l'œil : c'est « un coup du visuel » (Ubersfeld, 199). Car l'objet dont il est question était, bien sûr, de ladite couleur. Rouge sang-debœuf, couleur du sang, de la vie. Couleur voyante, terrible, cruelle et belle. Couleur qui entraîne l'œil, chaude et saillante. Couleur d'encre. Couleur du crime, de l'interdit, de la faute. $\mathrm{Au} \mathrm{XV}$ siècle, couleur divine, impériale, royale; au XIX siècle, couleur tout à la fois du juge, du forçat, du bourreau... et de la guillotine. Le rouge est en effet la couleur distinctive desdits bois de justice, décidée par le parlement de Paris en 1709 qui avait appliqué des règles identiques à l'instrument comme au bourreau, celui-ci devant par ailleurs en porter la marque jusque sur le bras : « La marque du bourreau devra être de couleur rouge, mais sera d'une couleur distincte si le bourreau porte un habit rouge. Il ne devra jamais la quitter, même les jours de fêtes » (Delarue, 46). Comme Hugo pourra 
le constater en exil à Guernesey, les consignes seront suivies à la lettre, même hors de l'hexagone : «Et il me montrait du doigt [...] un ensemble de charpentes ayant la couleur rougeâtre du sapin $[$...] on n'avait pas jugé à propos de le peindre couleur de fer » (CV, 1307). Ce que l'œil voit d'abord donc c'est de la couleur, l'empire de la couleur, celle qui pourrait être définie comme hyper-couleur, car fondamentalement la couleur entre toutes la plus vive, archétypale, la première des couleurs. Rouge! «Monsieur Victor Hugo voyait la guillotine de profil ; ce n'était pour lui qu'un poteau rouge $»^{5}$ : le visage qu'a la chose se devine donc d'abord, à sa couleur, ensuite, à sa forme. Rouge : la couleur s'annonce d'entrée de jeu. Le rouge est d'ailleurs à la mode: on met des chapeaux rouges, des gilets à la Robespierre, on se meuble en bois acajou. La couleur alors c'est bien ce par quoi la chose arrive, ce par quoi elle embraye l'imaginaire.

7 Or, il arrive à la guillotine de vouloir se faire attendre, voire désirer, et d'arriver sous forme de colis, enveloppée comme le serait un paquet cadeau, emballé comme le serait un jouet que l'on devinerait, à travers le papier entrouvert, à sa couleur :

Sur le débarcadère des douaniers ouvraient des colis, et à travers les ais des caisses entrebâillées, [...] on distinguait des objets étranges, deux longues solives peintes en rouge, une échelle peinte en rouge, un panier peint en rouge, une lourde traverse peinte en rouge dans laquelle semblait emboittée par un de ses côtés une lame épaisse et énorme de forme triangulaire. (CV, 830)

La foule est au rendez-vous, qui fixe du regard, comme fascinée, le colis-cadeau à peine débarqué, à peine déballé, essayant de deviner de quelle surprise il s'agit là. Elle ne va pas être déçue. Quand la couleur parle, elle est stupéfiante. Elle en peint la langue qui elle même ne peut que répéter qu'elle ne voit que du rouge, du rouge, du rouge, comme on ne voit que du feu. Le regard est comme happé, frappé, par ce qui se présente à lui, par cet objet en suspens, en train de se faire, en train d'apparaître. C'est exactement la même fascination que celle de ce petit enfant qui, chez Baudelaire, sur les injonctions de la Fée du joujou, choisit de s'emparer du joujou le plus beau, le plus cher, le plus voyant, le plus frais, le plus bizarre. On veut croire que ce joujou-là était une guillotine miniature et que c'est une des raisons et non des moindres, qui firent que la mère de l'enfant gourmand «se récria sur [son] indiscrétion et s'opposa obstinément à ce qu'[il] l'emportât » (Baudelaire, 582).

8 Quoi de plus beau, de plus cher, de plus voyant, de plus frais, de plus bizarre, en effet, qu'une guillotine? On pourrait dire de cet objet comme d'une peinture, que la guillotine a une efficacité picturale. Dame Guillotine sût en effet soigner son apparence et, de fait, son image s'installa bientôt effectivement dans la vie quotidienne des français, dans les assiettes et sur les tasses, aux oreilles des femmes sous forme de boucles d'oreilles en vermeil, ou encore servant d'instrument pour couper les fruits sur les tables... On ne s'étonnera pas d'apprendre alors que, très vite, la guillotine fut un jouet d'enfant. Au sens propre. C'est-à-dire qu'on récompensait les enfants sages par de petites guillotines. Il n'est pas jusqu'aux marionnettes elles-mêmes qui ne remplacèrent la potence par la guillotine! Or l'instrument de mort servait, bien sur, aussi de jouet aux adultes qui «tuaient leur temps auprès d'une petite guillotine en acajou qu'on apporte au dessert. On y fait passer successivement plusieurs poupées dont la tête [...] en tombant laisse sortir du corps, qui est un flacon, une liqueur rouge comme du sang. Tous les assistants, les femmes surtout, se hâtent de tremper leurs mouchoirs dans ce sang, qui se trouve être une eau ambrée très agréable $»^{6}$. On veut voir, on veut toucher la chose, à défaut d'y mettre son mouchoir, la main ou le cou, enfin, on voudrait bien savoir ce que ça fait. La curiosité est aiguisée et vient tempérer de gaité la sévérité qui 
semblait propre au cérémonial qui entourait la machine à décapiter. À tel point que jouer à la guillotine devient une pratique sociale, on voudrait pouvoir s'offrir la guillotine au dessert, comme la cerise sur le gâteau, et le rêve serait de pouvoir jouer à se faire guillotiner pour de vrai...

\section{Coup de théâtre}

Si Victor Hugo en vient à se dire que Paris n'est plus Paris puisqu'une Shéhérazade risque d'avoir le cou coupé tous les matins, on peut y voir là le risque terrible que représente l'instrument : c'est qu'à le voir, on ne peut plus se raconter d'histoires. Pour contrer la chose, l'écrivain se fera lui-même Shéhérazade, c'est-à-dire un raconteur d'histoires. Histoires de guillotine, s'entend. Le deuxième moment du coup de théâtre romanesque est celui qui essaye de voir comment fonctionne le choc du regard, non plus seulement à l'apparition de la chose seule ou de sa couleur, mais en racontant une scénette, en mettant les personnages et la chose - ou sa couleur - en contexte. De ce fait l'objet guillotine se retrouve non pas tant dans sa concrétude historique ou politique, que mis en fable dans le récit comme point de départ pour lancer diverses anecdotes, mini récits, histoires autour de l'objet en question, histoire toutes plus croustillantes les unes que les autres, bien sur. Telle celle de cette jeune fille, toute rose, nous précise Hugo, qui nous raconte qu'elle se fit expliquer par le bourreau, dans les moindres détails, ce qu'on appelle la toilette des condamnés. Et quand le bourreau lui explique que cela s'appelle enfourner, Hugo ne manque pas de noter ce qui aurait été la réponse de la jeune fille: «- Eh bien, Monsieur Sanson [...], je désire que vous m'enfourniez » (CV, 923).

Moment intense où la guillotine montre tout son pouvoir de séduction : la guillotine est un éblouissement et semble susciter de tout sujet qui s'en approche le désir de se faire enfourner. Le public de ces petites filles roses qui donne audience si attentive au bourreau Sanson nous l'indique, c'est leur télé-pathie, avide de goûter elle aussi au fantasme de cette singularité. La langue va droit au but: on enfourne, on fourre sans coup férir le premier corps qui se présente, tout cru. De fait, l'argot conte la chose, faisant croiser le sens propre et le figuré, comme l'écrivain croise lui-même l'anecdote racontée et le conte de fée pour enfant. On n'est pas loin de la scène du grand méchant loup. À ceci près que le petit chaperon n'est pas rouge mais rose, ayant l'unique désir, en somme, de vouloir se faire mettre le capuchon: en somme, nous dit l'écrivain, il fallut céder, c'est-à-dire la boucler avec la ceinture de cuir, la coucher sur la bascule, et refermer sur elle le capuchon de la lunette avant qu'elle ne se déclare satisfaite, au grand soulagement de Sanson qui avait peur qu'elle ne lui demande encore quelque chose...

11 Le tableau de jeunes filles renverse ainsi le choc visuel premier de l'instrument : c'est un tableau en mouvement (une jeune fille toute rose de curiosité) qui vient renverser le cérémonial établi (le rituel de la toilette des condamnés). La guillotine, toujours dressée, toujours érigée - rouge de désir, en somme - est ainsi prête à enfourner les corps de jeunes filles innocentes. Cérémonial sacrificiel, où le corps, une fois bouclé sur la bascule, ressemble à une bête donnée en offrande, pieds et poings liés. La scène est pour le moins ambiguë. Elle met en circulation les traverses des significations : la guillotine est à la fois l'instrument du jeu dont on peut jouer comme on joue du violon, l'instrument du désir et le couperet qui opère le passage de la vie à la mort, assisté par un guide. Ce n'est plus la chose même ou sa couleur qui créé le coup de théâtre, c'est 
son mécanisme que mime le mécanisme de l'anecdote : les étapes dudit enfournement nous sont contées par degrés. Seule la syntaxe marque les articulations par phrases courtes et sèches, répercutant l'angoisse, la difficulté de penser le fonctionnement de la chose jusqu'à la chute de son couteau. Or c'est tout de même par une chute, dans l'autre sens du terme, que se termine l'anecdote de Hugo, le bourreau se prenant à imaginer ce qu'il en aurait été s'il avait dû jouer la scène jusqu'au bout.

La guillotine fait désormais partie de la scène parisienne, de son quotidien, de son paysage. Paris a son bourreau comme il a son boulanger. On voudrait pouvoir se faire enfourner, histoire d'y être, d'en être, de faire partie, comme Polichinelle (qui partage lui aussi l'attention, nous rappelle Camille Desmoulin), de la grande comédie qui se joue dans les rues de Paris. Après l'Opéra, la guillotine. Car la guillotine, elle aussi, bien sûr, a sa mise en scène et on va à la guillotine comme on va au spectacle. C'est-à-dire, pour être plus exact, qu'assister aux exécutions est un devoir et que le spectacle de la guillotine devient littéralement le plus grand spectacle du monde : «Après ça nous irons à l'Opéra [...] Et puis nous irons voir guillotiner. Je vous ferai voir le bourreau. Il demeure rue des Marais. Monsieur Sanson. [...] Ah on s'amuse fameusement!» (Mis, 761). Et pour que tout se déroule comme prévu, pour que cela se fasse dans l'ordre, on répète la scène de l'exécution, telle celle de Louis Ulbach, dont Hugo vit la répétition en septembre 1827 et qui ne fut pas étrangère, semble-t-il, à la rédaction du Dernier jour d'un condamné.

13 On comprend alors que la guillotine ne fait plus peur : elle est rentrée dans l'économie du théâtre; on rit, on pleure, on joue tous les jours et la rue affiche complet. Théâtre qui devient d'ailleurs officiellement de la même couleur, la couleur rouge pourpre des rideaux de scène, des fauteuils de velours du théâtre et de l'opéra venant remplacer définitivement en France le traditionnel noir ou bleu nuit. Rouge pourpre qui deviendra ainsi en cosmétologie, rouge théâtre. Pas étonnant dès lors que, par répercussion, la guillotine, qui avait elle aussi ses habituées se vit surnommée du nom de théâtre rouge.

Hugo ne peut que constater la façon dont la machine de mort se transforme en une machine à rire, tout aussi mécanique : «le grand ressort du spectre rouge est cassé. Tout le monde le sait maintenant. L'épouvantail n'épouvante plus. Les oiseaux prennent des familiarités avec le mannequin, les stercoraires s'y posent, les bourgeois rient dessus » (Mis, 789). Phrase énigmatique du poète qui dérouta bien des critiques, mais où l'on devine, dans ce spectre rouge au ressort cassé, les montants fantomatiques de l'échafaud. La répétition de l'événement a transformé les corps en marionnettes ou en grands Guignols, la guillotine est devenue "du mécanique plaqué sur du vivant » (Bergson, 29), incitant à rire de la scène, faisant du moment de l'exécution une mécanique comique. Le spectre rouge de la guillotine ne fait donc plus peur aux enfants. On rit de la guillotine, on lui tire la langue, on se querellerait presque pour son panier, on lui donne des petits noms affectueux comme à une amoureuse: «On se montre la guillotine et l'on rit. On l'appelle de toute sorte de petits noms: - Fin de la soupe, - Grognon, - La mère au Bleu (au ciel) - La dernière bouchée, - etc., etc. Pour ne rien perdre de la chose, on escalade les murs, on se hisse aux balcons, on monte aux arbres, on se suspend aux grilles, on s'accroche aux cheminées " (Mis, 464). Ainsi, la guillotine a ses comédiens, ses danses, ses chansons : "Avant hier soir, 30 mars, un enfant de sept ans passait sur le boulevard en chantant avec mille gestes et mille contorsions folâtres: La tête tranchée / Et le poing coupé! Vengeons-nous ou 
mourrons!» (CV, 1029). Car les chansons sont « comme la guillotine, elles coupent indéfiniment, aujourd'hui cette tête-ci, demain celle-là. Ce n'est qu'une variante » (CV, 484). La guillotine semble en effet exciter l'appétit de ses spectateurs, le bourreau inclus, à tel point qu'elle introduit avec l'idée du spectacle à voir, une sorte d'avidité de la répétition qui fait que même si l'instant de la décapitation se fait en l'espace d'un clin d'œil, il ne s'agit plus seulement de voir, mais de voir encore, de voir toujours plus.

\section{Le combat de l'encre}

Mais qui veut perdre la tête pour avoir volé une pomme? Ou pour avoir dit trop haut le mot liberté ? Car le bruit de ce charivari infernal ferait presque oublier qu'il s'agit de la question de la peine de mort dont la guillotine fut le plus fidèle instrument. Pour dire sa pensée sur la peine de mort, c'est donc à la guillotine que l'écrivain va attacher sa verve, car c'est l'instrument lui-même qui l'intéresse et qu'il veut remettre à sa place. Le spectre de la guillotine, spectre de l'horreur, dans la fascination qu'il exerce sur Hugo lui-même, devient non seulement l'objet de remarques philosophiques et historiques, mais s'introduit dans l'œuvre romanesque et picturale où le $\mathrm{H}$ lacunaire et énigmatique éclaire de ténèbres l'œuvre et jette sur l'initiale du nom Hugo lui-même un éclairage singulier. Car Hugo ne se prive pas du jeu idéographique, mêlant ainsi l'initiale de son nom propre avec le «hiéroglyphe » de la guillotine. On ne peut alors que penser à ce testament exemplaire qu'est la conclusion, le coup de hache final, de Quatre-vingt treize, dans un jeu cruel à la fois philosophique et littéraire, jeu d'humour noir dans le rouge, qui consiste à terminer l'œuvre romanesque d'un $\mathrm{H}$ grandiose, à la fois engin de décapitation et signature.

16 Au reste, il faut alors bien considérer que la guillotine est l'irreprésentable même et, par suite, l'inénarrable même. Ce qui n'arrange rien ni personne. La guillotine reste en deçà de toutes les figurations, de toutes les narrations, de tous les scénarios. Elle provoque un choc, une secousse violente, qui ne ressemble en rien à un rêve ou à l'image que nous nous faisons du rêve, qui empêche même le rêve de se faire: Hugo nous invite ici à penser et non à rêver. La guillotine est pensée. Elle heurte l'imaginaire par le choc de sa couleur et fait son trou dans la langue. Quand le regard hallucine, c'est l'ouverture pensante qui fait en effet émerger le sujet endormi et lui fait se poser la question : Qu'est-ce que cette couleur? Qu'est-ce que cette image? Il faut alors prendre en compte «la dimension physique et éthique proprement philosophique de l'“hallucination" hugolienne (en grec, très rigoureusement le mot désigne un rapport à l'autre)» (Maurel, 49). Si la guillotine a ce pouvoir de faire halluciner, c'est qu'en désignant un rapport à l'autre, elle nous force à voir autrement - elle nous force à voir l'autre de l'être, l'autre je, elle est celle qui déstabilise l'être même. On hallucine parce qu'il y a subversion de la vision. La guillotine vient greffer son cadre sur le texte, vient le border, comme pour en marquer la frontière. Elle alimente le texte hugolien et devient sa première image, sa materia prima.

17 L'originalité de Hugo tient alors en cela qu'il enferme son lecteur dans un triple réseau. Triangulaire, de toute évidence. Il y a ainsi le choc visuel de l'inattendu de la chose, le retournement surprise de la fable qui engendre l'émotionnel, et - après coup - la tentative d'appréhender par la réflexion les raisons de cette violence émotionnelle :

-- Le gros monsieur : vous disiez donc que l'auteur en questionna de petits enfants.

Impossible madame. Quand on a fait cet ouvrage-là. Un roman atroce !... -- 
Quelqu'un : mais ce roman : dans quel but l'a-t-il fait? -- Le Poète élégiaque : est-ce que je sais moi? -- Le philosophe : à ce qu'il paraît, dans le but de concourir à l'abolition de la peine de mort. -- Le gros monsieur : une horreur vous dis-je ! -- Le chevalier : ah ça ! C'est donc un duel avec le bourreau? -- Le poète élégiaque : il en veut terriblement à la guillotine. -- Un monsieur maigre: Je vois cela d'ici : des déclamations. -- Le gros monsieur : point : il y a là à peine deux pages sur ce texte de la peine de mort. Tout le reste ce sont des sensations. (Hugo, Dernier Jour, 425)

Des sensations - car c'est en effet à «l'émotionnel [que Victor Hugo] unit un travail intellectuel, une réflexion, et une projection sur l'avenir immédiat » (Ubersfeld, 201). Oui, pour Hugo, la guillotine est tout simplement l'irreprésentable, l'inassimilable, l'innommable.

Seule l'écriture peut ainsi répondre à la fascination de l'objet: à celle qu'il nomme vindicte et qui revendique des corps humains et opère par le sang, l'écrivain oppose sa propre baguette, prend la revanche en prenant la plume et dévide un fil de couleur rouge à travers l'œuvre. À noter toutefois que l'engagement si ferme de l'auteur du Dernier Jour d'un condamné dans la lutte contre la peine de mort ne repose pas simplement sur un sentiment d'horreur devant la manière hideuse de décapiter, la froideur métallique, comme abstraite, géométrique, d'un usage monstrueux de l'angle d'attaque ou du triangle : l'hallucination et la répulsion devant le sang répandu est en effet inséparable d'une justification de pensée, d'une réflexion critique sur la société ou sur son ordre. Inséparable encore d'une remise en question politique et sociale de l'essence de la justice, dans une reprise profonde de l'idée de démocratie considérée comme le problème même de l'avenir humain, envisagé dans toutes ses dimensions, avec une lucidité et une largeur de vue que l'on doit qualifier de très rigoureusement philosophiques. Le rouge n'est alors plus seulement l'objet d'une détestation ou d'un dégoût, c'est un emblème.

On comprend alors mieux pourquoi la guillotine imprègne l'œuvre jusque dans ses retranchements : le papier en prend la couleur et on s'aperçoit tout à coup de « quelque chose de rouge qu'on a dans les ongles » (Mis, 969). D'un rouge qui fait étrangement écho aux billets écrits par les commissions d'exécuteurs décrits par Lenotre : «des billets écrits, après le travail, du bout de ces gros doigts qui avaient encore du sang aux ongles" (Lenotre, 7). Il semble que la main, quand elle est liée au crime, garde la couleur de son acte. Rouge, qui est signe d'exécution comme il est signe de bataille. Car, comme Hugo l'écrit ailleurs, il s'agit de rendre coup pour coup : «je me cramponnerai tour à tour à toutes vos libertés, et chaque fois qu'on m'en arrachera, j'en emporterai un morceau sanglant dans les ongles " $(\mathrm{CV}, 214)$. Voici donc comment cette rouge préférence permet de mieux comprendre la stratégie hugolienne de l'ironie, ce choix cynique du pire, qui permet à l'écrivain de s'affirmer buveur de sang.

Il est à noter que si, comme l'affirme Pierre Georgel, les dessins de Hugo peuvent être considérés comme «le prolongement de l'œuvre littéraire » (Georgel, 492), alors le rouge, quand il apparaît sur les dessins, n'en prend que plus de sens. Sur les frontispices du $\mathrm{Rhin}^{7}$ ou des Travailleurs de la $\mathrm{mer}^{8}$, sur les cartes de visite ou les cartes d'étrenne', ce qui apparaît au centre, le plus souvent en capitales rouges, c'est le nom de Hugo lui-même. Rouge Hugo, donc, placé au cœur de l'acte de peindre ou de dessiner comme la clef de son opération. Car si Hugo fait front à la guillotine et donc la peine de mort, c'est aussi en opposant son nom propre comme rempart. Il nous montre ainsi tout ce qui est à l'œuvre dans une couleur et que, pour en rendre compte, il faut compter avec lui. Ainsi, il ne faut pas éluder la puissance de la métaphore qui emporte, 
transfigure, et déplace sublimement cette image de la guillotine dans le texte, pour l'identifier avec l'initiale de l'auteur, qui apparaît dès lors comme le maître d'une contre-décapitation politique et existentielle. Que Hugo n'en finisse pas de jouer avec les mots mais aussi et surtout les noms propres, jusqu'à mettre en jeu son propre nom, c'est le signe même de cette liberté de l'écriture qui questionne, déplace, inquiète, et qui, contre toute attente, renverse les rôles, tout comme la posture de l'auteur.

Signer, saigner: ce mot d'ordre guide alors une exploration en quelque sorte physiologique, mais aussi bien « mot à mot », de l'œuvre. Car n'est-ce pas ce fil rouge de la signature qui permet de débusquer la lettre même d'un responsable? D'un signataire sur la brèche, décidément à vif? C'est à cet égard que le rouge est révélateur. Et peutêtre est-ce ainsi qu'il faut, désormais, considérer l'empreinte à vif de la figure hiératique statufiée du Grand Homme : comme prise et compromise avec ce fil rouge qui veut nouer indissociablement fibre vitale et fil du texte.

À l'éclair du triangle de la guillotine, répond ainsi l'éclair de la plume de l'écrivain et la guillotine ne prend tout son relief qu'à se baigner dans cette couleur particulière, où les mots sont écrits à la lumière de la chandelle même qui servirait à la suifer. Hugo notera alors dans ses carnets cette remarque qui lui fut faite « un soir de $18 \ldots$ » : «Vous n'avez pas lié les mains à la peine de mort, mais vous lui avez fait venir la rougeur au front » $(\mathrm{CV}, 1295)$.

\section{BIBLIOGRAPHIE}

Baudelaire, Charles. CEuvres Complètes. Paris : Gallimard, coll. « Bibliothèque de la Pléiade », 1975.

Bergson, Henri. Le Rire, Essai sur la signification du comique. coll. « Quadrige », Paris : PUF, 1940.

Boulard, Stéphanie. Rouge Hugo. Lille : PU du Septentrion, 2014.

Delarue, Jacques. Le Métier de bourreau. Paris : Fayard, 1979.

Georgel, Pierre. « Les Avatars du peintre malgré lui », La Gloire de Victor Hugo. Paris, Réunion des musées nationaux, 1986.

Hugo, Victor. Choses Vues, Euvres Complètes. Paris : Robert Laffont, 1987.

Hugo, Victor. Le Dernier Jour d'un condamné, Cuvres Complètes. Paris : Robert Laffont, 1985.

Hugo, Victor. Les Misérables, CEuvres Complètes. Paris : Robert Laffont, 1985.

Hugo, Victor. Les Travailleurs de la mer, Euvres Complètes. Paris : Robert Laffont, 1985.

Larousse, Pierre. «Guillotine ». Grand Dictionnaire Universel du XIX ${ }^{e}$ siècle. Genève : Slatkine, 1982.

Le Brun, Annie. Les Arcs-en-ciel du noir : Victor Hugo. Paris : Gallimard, 2012.

Lenotre G. La Guillotine et les exécuteurs des arrêts criminels pendant la révolution. Paris : Édition

Perrin, 1910,

Maurel, Jean. Victor Hugo philosophe. Paris : PUF, 1985. 
Millet, Claude. « Noir, blanc, couleurs », L'œil de Victor Hugo. dir. Guy Rosa et Nicole Savy, Paris :

Éd. des Cendres, 2004.

Milner, Max. «L'ombre qui donne à voir ", L'œil de Victor Hugo. dir. Guy Rosa et Nicole Savy, Paris : Éd. des Cendres, 2004.

Ubersfeld, Anne. «Le choc du regard ». L'œil de Victor Hugo. dir. Guy Rosa et Nicole Savy, Paris : Éd. des Cendres, 2004.

\section{NOTES}

1. « Regarder l'ombre, ce n'est pas regarder, c'est contempler» (Hugo, Les Travailleurs, 239).

2. Voir par exemple l'article de Max Milner, «L'ombre qui donne à voir », in L'œil de Victor Hugo, dir. Guy Rosa et Nicole Savy, Paris : Éd. des Cendres, 2004, 465.

3. Dans cet article, écrit à l'occasion du Colloque Annuel de la Société des Amis D'Inter-Textes ayant eu lieu à l'Université Sorbonne-Nouvelle Paris III en juin 2009, je tire ici le fil principal de la couleur, à vif. J'ai depuis exploré plus en détail tous ces aspects dans mon livre Rouge Hugo, publié en 2014.

4. Victor Hugo, Justitia, 1857. Plume, pinceau, encres brune et noire et lavis, crayon de graphite, fusain, rehauts de gouache rouge, réserves, zones frottées sur papier beige. Paris, Maison de Victor Hugo, Inv. 966. http://expositions.bnf.fr/hugo/grand/011bis.htm

5. C'est dans Victor Hugo raconté par un témoin de sa vie, qu'est racontée la scène de la montée à l'échafaud du parricide Jean Martin.

6. Grand Dictionnaire Universel $d u$ XIXe siècle par Pierre Larousse, article "Guillotine ", Réimpression de l'édition de Paris 1866-1879, Paris, Slatkine, 1982.

7. http://expositions.bnf.fr/hugo/grands/060.htm

8. http://expositions.bnf.fr/hugo/grands/246.htm

9. Voir par exemple cette carte d'étrennes du $1^{\mathrm{er}}$ janvier 1857: http://expositions.bnf.fr/hugo/ grands/156.htm

\section{RÉSUMÉS}

"Nous n'avons que le choix du noir », écrit Victor Hugo dans William Shakespeare. Le choix du noir, c'est bien celui en effet que fait Victor Hugo tant dans ses dessins que dans ses écrits, utilisant dans les deux cas la même plume, la même encre, voire le même papier, plaçant l'encre au cœur de la dialectique ombre/lumière. Nombre de critiques auront donc noté cette prédominance accordée à l'ombre et aux ténèbres pour y lire une phénoménologie négative visà-vis du monde visible, les autres couleurs venant nécessairement s'établir en contraste ou au contraire du noir.

Or, si dès la Préface de Cromwell Hugo sort la couleur de sa contingence et qu'il l'engage dans l'écriture qui vient ainsi faire un pendant aux dessins, ce n'est pas seulement pour en colorer son style, mais pour l'inscrire dans un système symbolique et métaphorique complexe. Pour Victor Hugo, en effet, l'idée $a$ une couleur et, particulièrement, l'idée de rouge. Nous voulons démontrer en effet que le rouge, chez Hugo, établit avec l'expérience de l'ombre une voie de passage ou une 
complémentarité. Le rouge envahit les pages de ses romans ou de ses cahiers de notes, et devient en tant que tel une vision haptique - spectacle de théâtre, pôle d'attraction, signe politique, entourant surtout un objet entre tous, un centre de fascination absolu et terrible : la guillotine. Quelle idée vient donc colorer de rouge l'écriture hugolienne? Quel acte métaphysique ou idéologique communique ou converge dans cette couleur?

"Black is the only choice we have," Victor Hugo writes in William Shakespeare. He chooses to use black as much in his drawings as he does in his writing, for in each place he uses the same pen, the same ink, and even the same paper, pouring ink into the heart of the dialectic between shadows and light. Many critics have thus noted this predominance given to darkness in Hugo's work, reading them as a negative phenomenology operating in relation to the visible world, as other colors appear simply in contrast or opposition to the color black.

And yet if beginning with the Préface de Cromwell, Hugo frees color from its contingency and engages it in writing that thus comes to balance his drawings this is not only to color his style from it, but in order to place it into a complex symbolic and metaphorical system. In fact, for Victor Hugo, ideas have a color, and particularly the idea of the color red. We wish to demonstrate that for Hugo this color red does not oppose his shadows, but rather creates a means of passage or complementarity with them. Red, almost absent in his drawings, paradoxically invades the pages of his novels and notebooks, becoming a truly haptic vision - a theatrical spectacle, a center of attraction, a political sign - surrounding one object, a center of absolute and terrifying fascination, over all others: the guillotine. What idea therefore colors Hugo's writing red? What metaphysical or ideological act converges or is communicated in this color?

\section{INDEX}

oeuvrecitee Dernier Jour d'un condamné (Le), Misérables (Les)

Keywords : guillotine, red, death penalty, red theater, Sanson

Mots-clés : guillotine, rouge, peine de mort, théâtre rouge, Sanson

\section{AUTEURS}

\section{STÉPHANIE BOULARD}

Stéphanie Boulard est Maître de Conférence en littérature française à l'Université de Georgia Tech (Atlanta, USA) et affiliée au Groupe Hugo-CERILAC (EA 4410). Sa recherche questionne les interactions entre la littérature et les arts visuels du XIX ${ }^{\mathrm{e}}$ siècle au contemporain. Auteur de Rouge Hugo (Presses du Septentrion, 2014), elle a aussi dirigé un numéro de la Revue des Sciences Humaines consacré à Victor Hugo (Ego Hugo, Presses Universitaires du Septentrion, 2011), ainsi qu'un livre sur l'œuvre de Claude Louis-Combet (Visions Visitations Passions : l'art de Claude-Louis Combet, Corlevour, 2008) et co-dirigé, avec Christophe Ippolito, un numéro spécial de la revue SITES portant sur l'œuvre de Pascal Quignard (SITES, 2014). Elle est l'auteur de plus de 20 articles portant entre autres sur les œuvres de Hugo, Balzac, Genet, Quignard, Cixous, Michaux, LouisCombet. 